\title{
Dynamic Characteristics of a YBCO Bulk above an Electromagnet Guideway
}

\author{
Chaoqun Zhao, Mark D. Ainslie, Senior Member, IEEE, Jing Li, Wei Hong and Ying Xin, Senior Member, IEEE
}

\begin{abstract}
For high-temperature superconducting (HTS) maglev, an electromagnet guideway unit (EMGU) that can form an electromagnet guideway (EMG) with a small gap, or even no gap, between EMGUs has been designed. The longitudinal magnetic fields along a single EMGU and two EMGUs arranged in a line were first investigated through measurement and simulation. The experimentally measured data validated the simulation results from a three-dimensional (3D) EMGU model implemented in COMSOL Multiphysics, indicating that the model is reliable and can be used for further studies. The dynamic responses of a high-temperature superconducting (HTS) bulk above a single EMGU and two EMGUs arranged in a line, including the dynamic levitation force (LF) and traveling directional force (TDF), under different operating conditions were investigated through experiment and simulation using a segregated $\boldsymbol{H}$ formulation model. The magnetic field and current density distributions inside the superconductor are affected by the external magnetic field generated by the EMGU and are responsible for the dynamic characteristics. Finally, the segmented instant excitation (SIE) mode was investigated through simulation, which shows it is feasible by coordinating the currents of EMGUs.
\end{abstract}

Index Terms-Electromagnet guideway, HTS maglev, dynamic levitation characteristics, bulk high-temperature superconductors, numerical modeling, HTS modeling.

\section{INTRODUCTION}

$\mathrm{S}_{\mathrm{se}}^{\mathrm{N}} \mathrm{sin}$ INCE the discovery of the HTS maglev phenomenon, researchers around the world have made significant efforts to develop HTS maglev using permanent magnet guideway (PMG) [1-3]. To date there has been considerable research on the performance of HTS-PM maglev system. The magnetic field distribution generated by different PM arrangements has been investigated [4-7] and the levitation force and guidance force of an HTS bulk over a PM have been studied to evaluate the HTSPM levitation performance [8-14]. Moreover, the dynamic properties of an HTS bulk over a PM has been investigated by experiments and simulations [15-17].

However, to the best of our knowledge, the levitation performance - in particular, the dynamic characteristics - of an HTS bulk above electromagnet guideway units, or EMGU(s), has not been studied in great detail. HTS maglev with an electromagnet guideway (EMG) will avoid the use of PMs (rare resources), especially if the guideway is installed over long distances. Unlike a PMG, the magnetic field generated by an EMG can be

This work was supported in part by the National Natural Science Foundation of China (Grant No. 51677131) and the Engineering and Physical Sciences Research Council under Grant EP/P020313/1. All data are provided in full in the results section of this paper. (Corresponding author: Ying Xin.)

Chaoqun Zhao, Wei Hong and Ying Xin are with the School of Electrical and Information Engineering, Tianjin University, Tianjin 300072, China. (e-mail: yingxin@tju.edu.cn). Jing Li is with the Renai College of Tianjin University, Tianjin 301636, China. regulated dynamically. In contrast to a PMG, the attraction of ferromagnetic materials is not an issue for the EMG when it is not energized, which increases the convenience of maintenance. In principle, on the premise of the superconductor having sufficient levitation force and guidance force, the EMG can adopt a segmented instant excitation (SIE) mode to achieve a minimum levitation power loss. The SIE mode is to energize the EMGUs below the position where superconductors reach, and reduce the current or even power off the EMGUs below the position where superconductors have travelled past [18-20].

In this paper, a comparative analysis of the longitudinal magnetic field generated by a single EMGU and two EMGUs arranged in a line was first carried out through measurement and simulation. The dynamic characteristics of a YBCO bulk above a single EMGU and two EMGUs arranged in a line, under different operating modes, were investigated through experiment and simulation. Finally, the SIE mode with levitation force control was investigated through simulation.

\section{EXPERIMENTS}

\section{A. Design of EMGU and Force Measurement Setup}

The specifications of a previous EMGU [21] were modified to enhance the magnetic field flux density for levitation performance. Fig. 1 shows the modified specifications of two same EMGUs (EMGU1 and EMGU2) arranged in a line. The levitation space is above the middle post. The two side posts of each

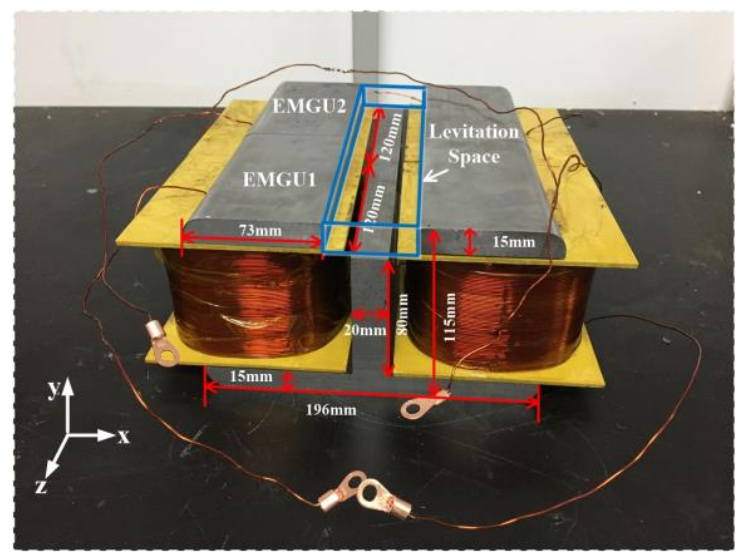

Fig. 1. Structure of two electromagnet guideway units (EMGUs) arranged in a line.

Mark D. Ainslie is with the Bulk Superconductivity Group, Department of Engineering, University of Cambridge, Trumpington Street, Cambridge CB2 1PZ, UK.

Color versions of one or more of the figures in this paper are available online at http://ieeexplore.ieee.org.

Digital Object Identifier will be inserted here upon acceptance. 


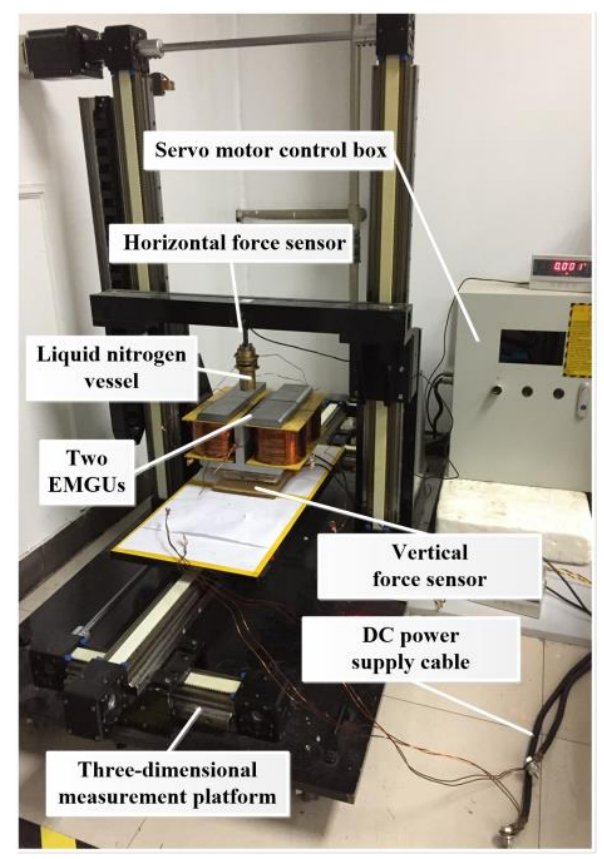

Fig. 2. The force measurement system.

EMGU are wrapped with two coils that are connected in series and the number of turns of each coil is 1495 . The dimensions of the side posts wound by these coils in the $x$ - and $z$-axis directions are both $47.5 \mathrm{~mm}$. The $z$ - and $x$-axis directions correspond to the longitudinal and transverse directions, respectively. The two EMGUs can be connected in series or in parallel as energized by the power supplies. Multiple EMGUs can form an EMG with a small gap or even no gap between them.

Fig. 2 shows our force measurement system that can provide three-dimensional movement at different velocities. The superconductor fixed inside the bottom of the liquid nitrogen vessel is a melt-textured YBCO bulk with a diameter of $30 \mathrm{~mm}$ and a thickness of $15 \mathrm{~mm}$, which was provided by the General Research Institute for Nonferrous Metals.

\section{B. Experimental Details}

The $y$-component of the magnetic flux density along z-axis, $B_{\mathrm{y}}(z)$, of a single EMGU1 and two EMGUs arranged in a line at a height of $7 \mathrm{~mm}$ above the center of guideway with DC currents of 1, 2, 3 and $4 \mathrm{~A}$ was measured.

The dynamic responses above a single EMGU energized by a power supply, and for two EMGUs, arranged in a line and individually energized by two power supplies, were investigated.

(1) Dynamic characteristics of the YBCO bulk above a single EMGU including two operating modes:

a) Current regulation mode for two cases

The YBCO bulk is first field-cooled in the magnetic field corresponding to a current of $2.5 \mathrm{~A}$ at a height of $7 \mathrm{~mm}$ (or at $27 \mathrm{~mm}$ and then the bulk descends to the height of $7 \mathrm{~mm}$ ). A set of current oscillations are then initiated at a rate of $0.5 \mathrm{~A} / \mathrm{s}$ following the cooling process. Firstly, the two oscillations of current are from $2.5 \mathrm{~A}$ to $4 \mathrm{~A}$, then to $1 \mathrm{~A}$, and back to $2.5 \mathrm{~A}$. The last oscillation is from $2.5 \mathrm{~A}$ to $4 \mathrm{~A}$ and then to $0.5 \mathrm{~A}$. During the current oscillations, the levitation force at intervals of $0.5 \mathrm{~A}$ is collected.

b) Both movement and current regulation modes for two cases

The bulk positioned at $z=-30 \mathrm{~mm}$ (the center of the EMGU is at $z=0 \mathrm{~mm}$ ) is field-cooled in the presence of the magnetic field corresponding to a current of $1 \mathrm{~A}$ at a height of $27 \mathrm{~mm}$, and then descends to a height of $7 \mathrm{~mm}$ at a velocity of $2 \mathrm{~mm} / \mathrm{s}$. Finally, the current varies at a rate of 1 A every 2 seconds or every 5 seconds from $1 \mathrm{~A}$ to $4 \mathrm{~A}$ and back to $1 \mathrm{~A}$, when the bulk moves to the position of $z=30 \mathrm{~mm}$ along the $z$-axis at velocities of 2 or $5 \mathrm{~mm} / \mathrm{s}$.

(2) Dynamic characteristics of the YBCO bulk above two EMGUs (EMGU1 and EMGU2), arranged in a line with a gap of $2 \mathrm{~mm}$ between them:

The YBCO bulk is field-cooled in the presence of the magnetic field corresponding to currents of $3 \mathrm{~A}$ in both EMGUs, as well as the case of $1 \mathrm{~A}$ in EMGU1 and $3 \mathrm{~A}$ in EMGU2, at a height of $27 \mathrm{~mm}$ above the position of $z=-60 \mathrm{~mm}$ (the center of the gap is at $z=0 \mathrm{~mm}$ ). The bulk then descends to a height of $7 \mathrm{~mm}$ and moves to the position of $z=60 \mathrm{~mm}$ along the $z-$ axis. The descending speed and forward travelling speed of the YBCO bulk are $2 \mathrm{~mm} / \mathrm{s}$ and $5 \mathrm{~mm} / \mathrm{s}$, respectively.

\section{NUMERICAL MODEL}

Numerical modelling is a powerful tool to estimate HTS levitation performance. Some models simplified the real geometry to $2 \mathrm{D}$ by considering the guideway and superconductor to be infinitely long, which qualitatively described the electromagnetic behavior and basic properties [22, 23]. However, a more realistic 3D model can deal with complex operating conditions, which cannot be tackled by a 2D model. The $\boldsymbol{H}$-formulation has been applied to analyse various problems related to HTS materials [24-29]. In this study, a 3D HTS-EMGU model is established in COMSOL Multiphysics using a segregated $\boldsymbol{H}$-formulation method to simulate the dynamic responses [9, 30-32]. The finite-element model consists of separate (segregated) EMGU and HTS models. The coupling between these two models is achieved by applying the sum of the external magnetic field of the EMGU and the self-field of the HTS bulk on the outer boundaries of the HTS model, which consists of an HTS subdomain surrounded by a thin air subdomain. The selffield generated by superconductor is calculated from the numerical integration of the Biot-Savart law.

The EMGU model is a magnetostatic finite-element model of the EMGU energized by a DC current, which is used for the analysis of the magnetic field generated by the EMGU. The simulation results of the longitudinal magnetic field are compared with measured data obtained from the experiments detailed in Section II.B as shown in Fig. 3. In addition, in order to investigate the dynamic characteristics of YBCO bulk above two EMGUs, the $B_{\mathrm{y}}(z)$, at a height of $7 \mathrm{~mm}$, generated by a DC currents of $3 \mathrm{~A}$ in both EMGUs, as well as the case of a DC current of $1 \mathrm{~A}$ in EMGU1 and $3 \mathrm{~A}$ in EMGU2, with a gap of 2 $\mathrm{mm}$ between the EMGUs are simulated, as shown in Fig. 4.

The HTS model is time-dependent and modelled using the $\boldsymbol{H}$-formulation that is derived from Maxwell's equations and represented in its general form by, 


$$
\mu_{0} \frac{\partial \boldsymbol{H}}{\partial t}+\nabla \times(\rho \nabla \times \boldsymbol{H})=0
$$

where $\boldsymbol{H}$ represents the magnetic field strength and $\rho$ is the resistivity. The nonlinear resistivity of superconductor is represented by the $E-J$ power law:

$$
\rho(\boldsymbol{J})=\frac{E_{C}}{J_{C}(\boldsymbol{B})}\left|\frac{\boldsymbol{J}}{J_{C}(\boldsymbol{B})}\right|^{n-1}
$$

where $\boldsymbol{J}$ is the current density; $J_{C}$ is critical current density; $E_{C}$ is critical current characteristic electric field. A field-dependent critical current density, $J_{C}(\boldsymbol{B})$, is considered in this simulation using the Kim-like model [33],

$$
J_{C}(\boldsymbol{B})=J_{c 0} \frac{B_{0}}{|\boldsymbol{B}|+B_{0}}
$$

where $J_{c 0}$ the self-field critical current density and $B_{0}$ is a material parameter. The isotropic assumption was considered in HTS model for simplicity. The resistivity of the air subdomain is assumed simply as $1 \Omega \mathrm{m}$. All of the parameters assumed for the EMGU and HTS models are listed in Table I.

The external magnetic field relative to the position of the HTS bulk is realized by a spatial function that can indicate the position of HTS domain. We need a dynamic magnetic field to simulate the current regulation of EMGU(s). Therefore, we first obtain static external unit magnetic field generated by current of 1 A of the EMGU(s), and then the magnetic field is multiplied by a time-dependent function in HTS model. The timedependent function will be detailed in Section IV.A.

The levitation force (LF) and travelling directional force (TDF), respectively, are calculated by:

$$
\begin{aligned}
L F(t)= & \iiint_{\Omega_{s c}} J_{z}\left(x^{\prime}, y^{\prime}, z^{\prime}, t\right) \cdot B_{x}\left(x^{\prime}, y^{\prime}, z^{\prime}, t\right)- \\
& J_{x}\left(x^{\prime}, y^{\prime}, z^{\prime}, t\right) \cdot B_{z}\left(x^{\prime}, y^{\prime}, z^{\prime}, t\right) d x^{\prime} d y^{\prime} d z^{\prime} \\
T D F(t)= & \iiint_{\Omega_{s c}} J_{y}\left(x^{\prime}, y^{\prime}, z^{\prime}, t\right) \cdot B_{x}\left(x^{\prime}, y^{\prime}, z^{\prime}, t\right)- \\
& J_{x}\left(x^{\prime}, y^{\prime}, z^{\prime}, t\right) \cdot B_{y}\left(x^{\prime}, y^{\prime}, z^{\prime}, t\right) d x^{\prime} d y^{\prime} d z^{\prime}
\end{aligned}
$$

where $\Omega_{s c}$ is HTS domain.

\section{RESULTS AND DisCUSSIONS}

\section{A. Longitudinal Magnetic Field}

Fig. 3 shows measured values (symbols) and simulation results (lines) of the $B_{\mathrm{y}}(z)$ with different currents (1, 2, 3 and $4 \mathrm{~A}$ ) at a height of $7 \mathrm{~mm}$ above the guideway surface, which show good agreement. The abbreviations "sim" and "exp" used in the figures refer to the simulation results and measured values, respectively. The measured values are from different $\mathrm{z}$ coordinates above a single EMGU1, as well as two EMGUs arranged in a line. The magnetic flux density of the newly designed EMGU was enhanced compared with that of our previous

TABLE I

\begin{tabular}{c|llc}
\multicolumn{4}{c}{ ASSUMED PARAMETERS FOR THE EMGU AND HTS MODELS } \\
\hline \hline Model & Symbol & \multicolumn{1}{c}{ Quantity } & Value \\
\hline & $E_{C}$ & $\begin{array}{l}\text { Critical current charac- } \\
\text { teristic electric field }\end{array}$ & $1 \times 10^{-4} \mathrm{~V} / \mathrm{m}$ \\
HTS & & $n$ value & 21 \\
model & $n$ & Kim model parameter & $5 \times 10^{7} \mathrm{~A} / \mathrm{m}^{2}$ \\
& $J_{c 0}$ & Kim model parameter & $0.37 \mathrm{~T}$ \\
& $B_{0}$ & Air/HTS permeability & $4 \pi \times 10^{-7} \mathrm{H} / \mathrm{m}$ \\
\hline $\begin{array}{c}\text { EMGU } \\
\text { model }\end{array}$ & $\mu_{0}$ & Number of turns & 1495 \\
\hline
\end{tabular}

design [21]. It can be seen from Fig. 3 that the magnetic field (external field) generated by a single EMGU increases nonlinearly with increasing current due to saturation of the iron core. If the magnetic field generated by a single EMGU1 with a current of $1 \mathrm{~A}$ is normalized to 1 , the magnetic fields generated by 2, 3 and $4 \mathrm{~A}$ are 2, 2.9, and 3.57, respectively. Therefore, the time-dependent function mentioned in section III can be approximated as a piecewise linear function. It can be found that the two EMGUs arranged in a line has a larger $B_{\mathrm{y}}(z)$ than that for a single EMGU with the same current. This phenomenon is more obvious as current increases.

From the solid green line in Fig. 4, a notch appears in the longitudinal magnetic field at the gap generated by currents of $3 \mathrm{~A}$ in both EMGU1 and EMGU2, indicating the EMGUs should be arranged as close as possible for a flat magnetic field. It can be seen from the solid blue line that the magnetic field transitions from the magnetic field of EMGU2 with a current of 3 A to that of EMGU1 with a current of $1 \mathrm{~A}$.

\section{B. Dynamic Characteristics above a Single EMGU for Two Operating Modes}

\section{1) Current Regulation Mode}

Fig. 5 shows the time-dependent function used in the HTS model for simulating current regulation. Fig. 6 shows the dynamic LF versus current oscillation with and without the descending process. The initial LF values were $6.6 \mathrm{~N}$ and $0 \mathrm{~N}$, respectively, for the initial current of $2.5 \mathrm{~A}$. During the current oscillation, the LF with the descending process has a larger range of adjustment than that without the descending process. This indicated achieving a certain LF is necessary for a larger adjustment range before current regulation, otherwise the LF

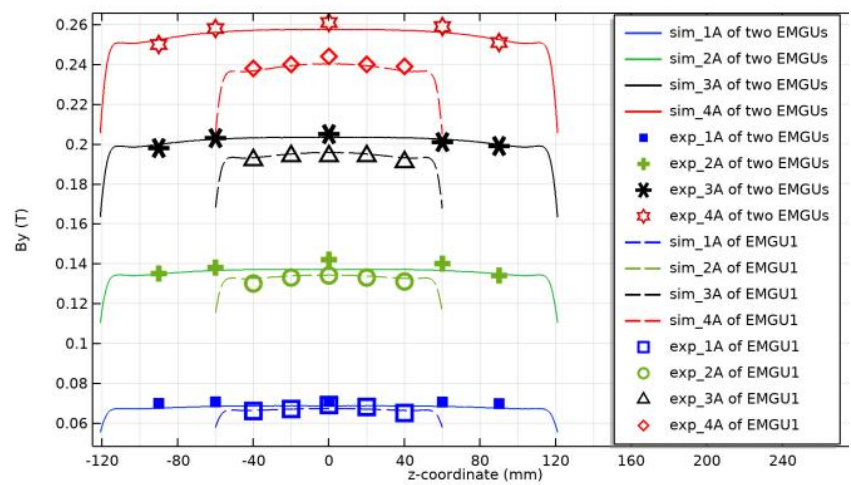

Fig. 3. The $B_{\mathrm{y}}(z)$ of a single EMGU1 and two EMGUs arranged in a line generated by different currents at a height of $7 \mathrm{~mm}$.

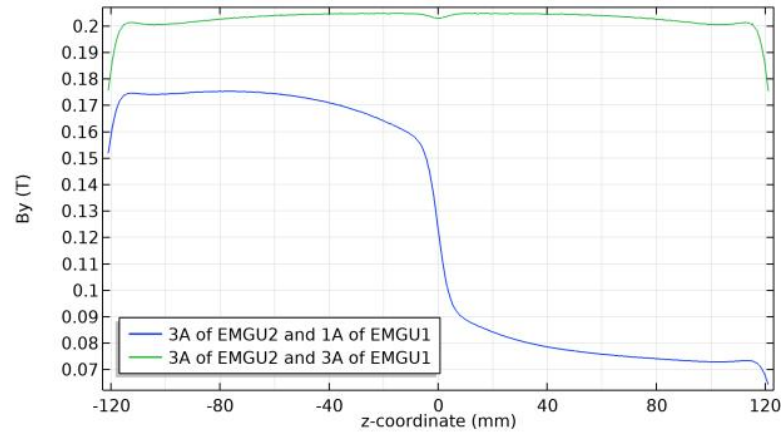

Fig. 4. Simulation of the $B_{\mathrm{y}}(z)$ generated by a DC currents of $3 \mathrm{~A}$ in both EMGUs, as well as the case of $1 \mathrm{~A}$ in EMGU1 and $3 \mathrm{~A}$ in EMGU2. 
(repulsive force) will be lost once the current is adjusted to lower than a current when superconductor is cooled down.

2) Forward Movement and Current Regulation Mode

Fig. 7 presents dynamic LFs with different forward velocities during the current regulation. The time-dependent function is also used in this simulation. For comparison, the maximum LFs in each case are shown together. The LF can be adjusted by varying the current during the movement of the superconductor above the EMGU. However, note that the LF values are different during ascent and descent, even with the same current.

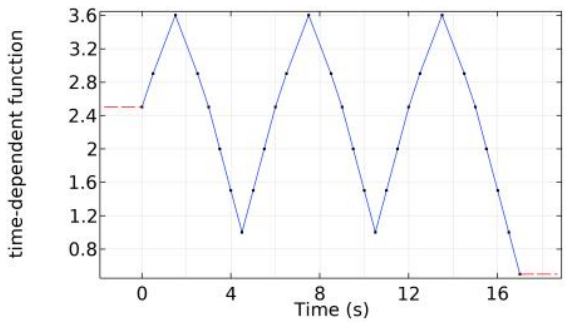

(a)

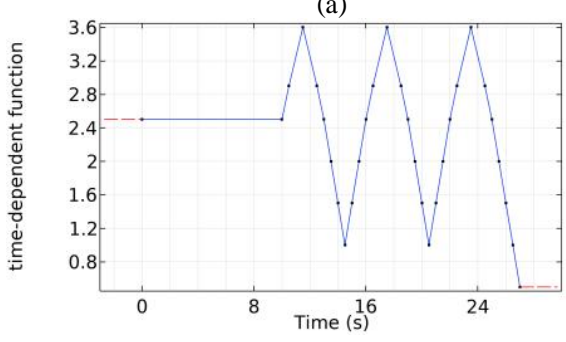

(b)

Fig. 5. Time-dependent functions used in the HTS model for simulating current regulation (a) without and (b) with descending process.

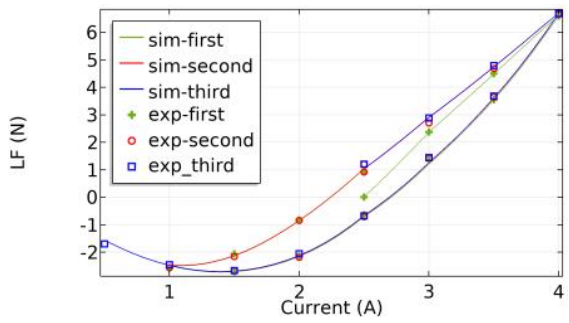

(a)

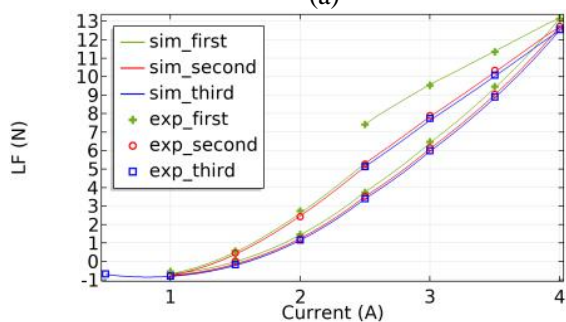

(b)

Fig. 6. LF versus current oscillation (a) without and (b) with descending process.

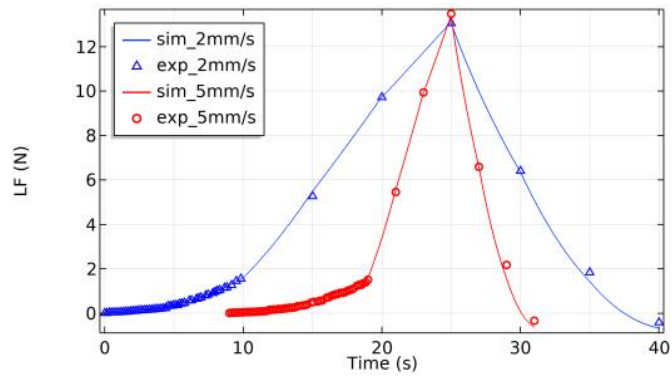

Fig. 7. Dynamic LF for different velocities with current regulation.

\section{Dynamic Characteristics above Two EMGUs}

Fig. 8 presents the dynamic LF and TDF values for a gap of $2 \mathrm{~mm}$ between EMGU1 and EMGU2. In Fig. 8(a), the currents in EMGU1 and EMGU2 are both $3 \mathrm{~A}$, whereas in Fig. 8(b) the currents are $1 \mathrm{~A}$ and $3 \mathrm{~A}$, respectively. It can be seen from Fig. 8(a) that the LF and TDF values have a small fluctuation when the YBCO bulk crosses the gap (from about 19 to $25 \mathrm{~s}$ ). This is because YBCO bulk experiences a reduced external magnetic field around the gap, which was shown in Fig. 4 (solid green line). This indicates that if the gap is small enough the bulk can move smoothly above the two EMGUs energized with the same current.

The LF value in Fig. 8(b) is $8.3 \mathrm{~N}$ at $t=10 \mathrm{~s}$, while it is 10.1 $\mathrm{N}$ in Fig. 8(a). This is because the external magnetic field generated by the currents of $3 \mathrm{~A}$ and $1 \mathrm{~A}$ is smaller than that when both currents are $3 \mathrm{~A}$, which can be seen from Fig. 4 . When the YBCO bulk moves from $z=-60 \mathrm{~mm}$ to $z=-25 \mathrm{~mm}$ above EMGU2 (around $t=10$ to $17 \mathrm{~s}$ ), there is a decrease in the LF and a little increase in the TDF. The decrease in the LF is because the external magnetic field (see blue line in Fig. 4) from $z=-75 \mathrm{~mm}$ to $z=-10 \mathrm{~mm}$ (considering the $30 \mathrm{~mm}$ diameter of the bulk) decreases a little. The decrease of the external field results in a new induced current, $i_{\text {ind2 }}$, whose direction opposes the induced current $i_{\text {ind1 }}$ obtained from descending process. $i_{\text {ind2 }}$ and the magnetic field inside the superconductor contribute to the decrease of the LF. The little increase in the TDF is because the magnetic field where the back half of the bulk is located is slightly larger than where the front half of the bulk located, which results in a slightly larger force experienced by the back half of the bulk in the positive $z$-axis direction in comparison to front half of the bulk in the negative $z$-axis direction. When the bulk moves from $z=-25 \mathrm{~mm}$ to $\mathrm{z}=25 \mathrm{~mm}$ (around $t=17$ to 27 s), LF decreases rapidly while TDF first increases then decreases. Here the reason for the decrease in LF is same as that around $t=10$ to $17 \mathrm{~s}$, but the external field decreases more

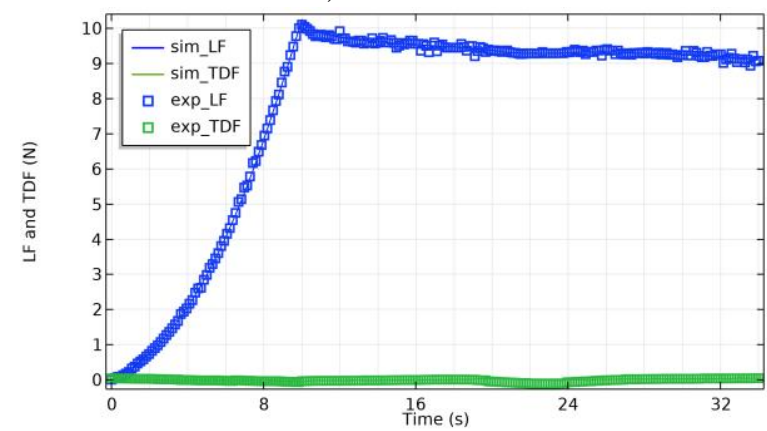

(a)

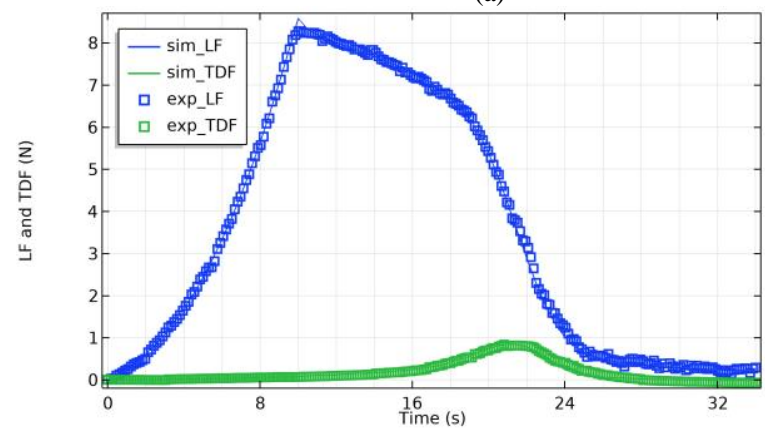

(b)

Fig. 8. Dynamic LF and TDF values for a gap of $2 \mathrm{~mm}$ and (a) current of $3 \mathrm{~A}$ in both EMGUs and (b) $1 \mathrm{~A}$ in EMGU1 and $3 \mathrm{~A}$ in EMGU2. 


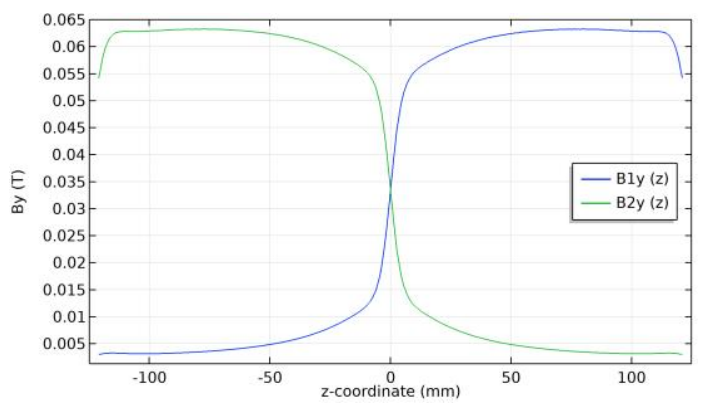

Fig. 9. Unit magnetic field $\mathrm{B}_{1 y}(z)$ and $\mathrm{B}_{2 y}(z)$.

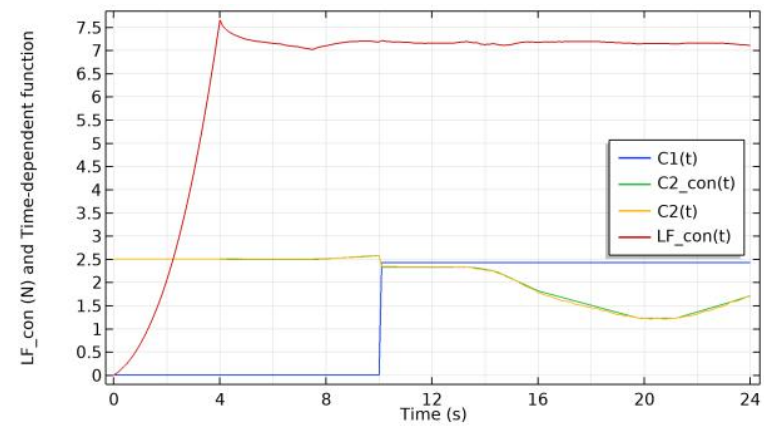

(a)

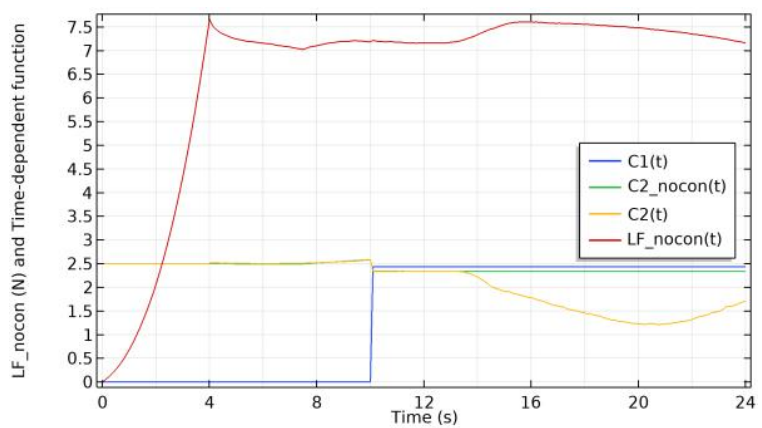

(b)

Fig. 10. $L F_{-}$con $(t)$ and $L F_{-n o c o n}(t)$ under the coordination of (a) $C_{1}(t)$ and $C_{2 \_c o n}(t)$ (b) $C_{1}(t)$ and $C_{2 \_n o c o n}(t)$, respectively.

rapidly. The reason for the increase in TDF here is the same as that for around $t=10$ to $17 \mathrm{~s}$. But the TDF here having a larger increase is because the magnetic field where the back half of the bulk located is much larger than that where the front half of the bulk located, as bulk moves from above EMGU2 to above the gap. As bulk moves from above the gap to above EMGU1, the force for the back half of the bulk in the positive $z$-axis direction decreases, while the force for the front half of the bulk in the negative $z$-axis direction changes little. Therefore, the TDF gradually increases as the YBCO bulk moves from about $t=17$ to $22 \mathrm{~s}$ (just above the gap) and decreases as bulk moves from about $t=22$ to $27 \mathrm{~s}$. When the bulk moves from $z=25$ $\mathrm{mm}$ to $z=60 \mathrm{~mm}$ (around $t=27$ to $34 \mathrm{~s}$ ), the LF and TDF decreases a little because of the slight decrease of magnetic field (see blue line in Fig. 4).

\section{The Investigation of SIE Mode}

In addition, for maintaining the LF value, the SIE mode of two EMGUs with a gap of $2 \mathrm{~mm}$ has been investigated through simulation. In this case, the HTS bulk positioned at $z=-100$ $\mathrm{mm}$ descends from a height of $27 \mathrm{~mm}$ to a height of $7 \mathrm{~mm}$ with EMGU2 energized. The bulk then moves along the two EMGUs from $z=-100$ to $100 \mathrm{~mm}$. The descending and moving speeds are both $5 \mathrm{~mm} / \mathrm{s}$.

In order to keep LF constant as the HTS bulk moves along the guideway, the $B_{\mathrm{y}}(z)$ constant, $B_{y_{-} \text {constant }}$, provided to superconductor is realized by the coordination of the real-time spatial magnetic field. Both the real-time spatial magnetic fields are obtained from the unit magnetic field multiplied by a time-dependent function. The unit magnetic field, $B_{1 y}(z)$, generated by currents of 0 and $1 \mathrm{~A}$, as well as $B_{2 y}(z)$ generated by currents of 1 and $0 \mathrm{~A}$, in EMGU2 and EMGU1, respectively, are obtained by implementing the model of the two EMGUs arranged in a line. These unit magnetic fields are shown in Fig. 9. In other words, $B_{y_{-} \text {constant }}$ is realized by the coordination of the two EMGUs, such that:

$$
C_{1}(t) B_{1 y}(z)+C_{2}(t) B_{2 y}(z)=B_{y_{-} \text {constant }}
$$

Changes in $C_{1}(t)$ and $C_{2}(t)$ represent the adjustment of the magnetic field of EMGU1 and EMGU2, respectively.

A $B_{y_{\_} \text {constant }}$ value of $0.158 \mathrm{~T}$ where the bulk descends, generated by currents of $3 \mathrm{~A}$ and $0 \mathrm{~A}$ in EMGU2 and EMGU1, respectively, is used. When $C_{1}(t)$ is set to 0 from $t=0 \mathrm{~s}$ to $10 \mathrm{~s}$ and to 2.43 from $t=10.1 \mathrm{~s}$ to $24 \mathrm{~s}, C_{2}(t)$ can be obtained from equation (6). $C_{2}(t)$ is an objective function for maintaining $B_{y_{-} \text {constant }}$. Fig. 10 shows $L F_{-}$con $(t)$ and $L F_{-}$nocon $(t)$ coordinated by the two EMGUs. To simplify things here, a $C_{2 \_c o n}(t)$ basically consistent with $C_{2}(t)$ is used in the simulation. $C_{2 \_ \text {con }}(t)$ is a function that traces the $C_{2}(t)$. To verify the role of the control method, $C_{2 \_n o c o n}(t)$ - without tracing $C_{2}(t)-$ is randomly set. $L F_{\text {ccon }}(t)$ is clearly more stable than $L F_{\text {nocon }}(t)$ as the bulk moves along the guideway. Where $C_{2 \_c o n}(t)$ in Fig. 10 (a) is decreasing from about $t=13$ to $20 \mathrm{~s}$ corresponds to the current of EMGU2 decreasing, which indicates that power is saved while the LF remains constant. Therefore, by controlling the current in each EMGU appropriately in the SIE mode, stable levitation force control can be achieved and the power dissipated in each EMGU can be optimized.

\section{CONClusion}

In this paper, the dynamic characteristics of a YBCO bulk above an electromagnet guideway (EMG) were investigated through experimental measurements and simulations using a $3 \mathrm{D}$ segregated $H$-formulation model. The simulation results reflect the behavior of the experimental dynamic characteristics well, both qualitatively and quantitatively. The 3D model can be used to analyze the electromagnetic interaction between the bulk and the EMG during the complex combination of the 
motion of the bulk in a 3D space and magnetic field regulation by varying the current.

A comparative analysis of the longitudinal magnetic field generated by a single electromagnet guideway unit (EMGU) and two EMGUs arranged in a line was first carried out through measurement and simulation, validating the model and indicating that the longitudinal magnetic field along the two EMGUs arranged in a line is larger than that of a single EMGU.

Achieving a certain levitation force (LF) value is necessary for a large enough range of adjustment before current regulation. Otherwise, the LF will be lost once adjusting the current lower than the current when superconductor is first cooled down. The LF is stable when superconductor moves above the two EMGUs (EMGU1 and EMGU2) with the same current and regulation of LF can be achieved by presetting different excitation conditions for the EMGUs as bulk moves along the guideway. The mechanism of these dynamic levitation characteristics is due to the electromagnetic interaction between the EMGUs and the bulk. The magnetic field and the current density distributions inside the superconductor are affected by the external magnetic field generated by EMGU and are responsible for the dynamic characteristics. Finally, the segmented instant excitation (SIE) mode to control the levitation force is shown to be feasible by coordinating the currents of EMGUs.

\section{REFERENCES}

[1] J. Wang et al., "The first man-loading high temperature superconducting maglev test vehicle in the world," Physica C, vol. 378-381, pp. 809-814, Oct. 2002.

[2] L. Schultz et al., "Superconductively levitated transport system-The SupraTrans project," IEEE Trans. Appl. Supercond., vol. 15, no. 2, pp. 2301-2305, Jun. 2005.

[3] G. G. Sotelo et al., "A full scale superconducting magnetic levitation (MagLev) vehicle operational Line," IEEE Trans. Appl. Supercond., vol. 25, no. 3, Art. no. 3601005, Jun. 2015.

[4] K. Ozturk, M. Abdioglu, E. Sahin, S. Celik, H. Gedikli and B. Savaskan, "The Effect of Magnetic Field Distribution and Pole Array on the Vertical Levitation Force Properties of HTS Maglev Systems," IEEE Trans. Appl. Supercond., vol. 25, no. 4, pp. 1-7, Aug. 2015.

[5] H. Ueda and A. Ishiyama, "Dynamic characteristics and finite element analysis of a magnetic levitation system using a YBCO bulk superconductor," Supercond. Sci. Technol., vol. 17, pp. S170-S175, Feb. 2004.

[6] Z. Deng, J. Wang, J. Zheng, Y. Lu, L. Liu, and G. Ma et al., "High-efficiency and low-cost permanent magnet guideway consideration for high$\mathrm{T}_{\mathrm{c}}$ superconducting maglev vehicle practical application," Supercond. Sci. Technol., vol. 21, no. 11, Art no.115018, Oct. 2008.

[7] N. Del-Valle, A. Sanchez, E. Pardo, D.-X. Chen and C. Navau, "Optimizing levitation force and stability in superconducting levitation translational symmetry", Appl. Phys. Lett., vol. 90, no. 4, Art. no. 042503 Jan. 2007.

[8] F. C. Moon, M. M. Yanoviak and R. Ware, "Hysteretic levitation forces in superconducting ceramics," Appl. Phys. Lett, vol. 52, no. 18 p. 1534, Mar. 1988.

[9] F. Sass, G. G. Sotelo, R. D. Andrade and F. Sirois, "H-formulation for simulating levitation forces acting on HTS bulks and stacks of $2 \mathrm{G}$ coated conductors", Supercond. Sci. Technol., vol. 28, no. 12, Art no. 125012, Nov. 2015.

[10] H. Li et al., "Lateral motion stability of high-temperature superconducting maglev systems derived from a nonlinear guidance force hysteretic model", Supercond. Sci. Technol., vol. 31, no. 7, Art no. 075010, Jun. 2018.

[11] G. Ma, J. Wang and S. Wang, "Numerical Investigation of the Lateral Movement Influence on the Levitation Force of the Bulk HTS Based on a 3-D Model," IEEE Trans. Appl. Supercond., vol. 20, no. 3, pp. 924-928, Jun. 2010.
[12] J. Zheng et al., "Magnetic and levitation characteristics of bulk high temperature superconducting magnets above a permanent magnet guideway," Supercond. Sci. Technol., vol. 29, no. 9, Art. no.095009, Jul 2016.

[13] N. Del-Valle, A. Sanchez, C. Navau, and D.-X. Chen, "Lateral-displacement influence on the levitation force in a superconducting system with translational symmetry", Appl. Phys. Lett., vol. 92, no. 4, Art no. 042505, Jan 2008

[14] H. Song et al., "Influence of the lateral movement on the levitation and guidance force in the high-temperature superconductor Maglev system," Appl. Phys. Lett., vol. 86, no. 19, Art no. 192506, May 2005.

[15] L. Liu et al., "Levitation force transition of high-Tc superconducting bulk superconductors within a maglev vehicle system under different dynamic operation," IEEE Trans. Appl. Supercond., vol. 21, no. 3, pp. 1547-1550, Jun. 2011

[16] H. Liao et al., "Dynamic levitation performance of Gd-Ba-Cu-O and $\mathrm{YBa}-$ $\mathrm{Cu}-\mathrm{O}$ bulk superconductors under a varying external magnetic field," $\mathrm{Su}$ percond. Sci. Technol., vol. 31, Art. no. 035010, Feb. 2018.

[17] H. Ueda and A. Ishiyama, "Dynamic characteristics and finite element analysis of a magnetic levitation system using a YBCO bulk superconductor," Supercond. Sci. Technol., vol. 17, no. 5, p. S170, Feb. 2004.

[18] C. Zhao, Y. Xin, W. Hong, Y. Wen, J. Li and H. Jin, "Magnetic Levitation Characteristics of HTS Bulk above Electromagnets", IEEE Trans. Appl. Supercond., vol. 30, no. 4, Art no. 3600905, Jun. 2020.

[19] Y. Wen, Y. Xin, W. Hong, C. Zhao, W. Li, "Comparative study between electromagnet and permanent magnet rails for HTS maglev", Supercond Sci. Technol. vol. 33, no. 3, Art no. 035011, Feb. 2020.

[20] W. Hong, Y. Xin, C. Wang, Y. Wen, C. Zhao, W. Li, "Technical Feasibility Study of an E-Shaped Electromagnetic Guideway for HTS Maglev”, IEEE Trans. Appl. Supercond., vol. 30, no. 6, pp. 1-10, Sept. 2020.

[21] C. Zhao, M. D. Ainslie, and Y. Xin, "Electromagnetic and Levitation Characteristics of a High-Temperature Superconducting Bulk above an Electromagnet Guideway," IEEE Access, vol. 8, pp. 195425-195435, 2020

[22] H. Liao, J. Zheng, H. Huang and Z. Deng, "Simulation and Experiment Research on the Dynamic Levitation Force of Bulk Superconductors Under a Varying External Magnetic Field," IEEE Trans. Appl. Supercond., vol. 29, no. 3, Art no. 3600805, Apr. 2019.

[23] J. Zheng, H. Huang, S. Zhang and Z. Deng, "A General Method to Simulate the Electromagnetic Characteristics of HTS Maglev Systems by Finite Element Software," IEEE Trans. Appl. Supercond., vol. 28, no. 5, Art no. 3600808 , Aug. 2018.

[24] K. Kajikawa, T. Hayashi, R. Yoshida, M. Iwakuma and K. Funaki, "Numerical evaluation of AC losses in HTS wires with 2D FEM formulated by self-magnetic field," IEEE Trans. Appl. Supercond., vol. 13, no. 2, pp. 3630-3633, Jun. 2003.

[25] Z. Hong, A. M. Campbell and T. A. Coombs, "Numerical solution of critical state in superconductivity by finite element software", Supercond. Sci. Technol., vol. 19, no. 12, pp. 1246-1252, Oct. 2006.

[26] R. Brambilla, F. Grilli and L. Martini, "Development of an edge-element model for AC loss computation of high-temperature superconductors", Supercond. Sci. Technol., vol. 20, no. 1, pp. 16-24, Nov. 2007.

[27] M. D. Ainslie et al., "An improved FEM model for computing transport AC loss in coils made of RABiTS YBCO coated conductors for electric machines", Supercond. Sci. Technol.vol. 24, no. 4, Art. no. 045005, Jan. 2011.

[28] M. D. Ainslie, W. Yuan and T. J. Flack, "Numerical Analysis of AC Loss Reduction in HTS Superconducting Coils Using Magnetic Materials to Divert Flux," IEEE Trans. Appl. Supercond., vol. 23, no. 3, Art. no. 4700104, Jun. 2013.

[29] V. Zermeno, F. Grilli and F. Sirois, "A full 3-D time-dependent electromagnetic model for Roebel cables", Supercond. Sci. Technol., vol. 26, no. 5, Art. no. 052001, May 2013.

[30] L. Queval et al., "Superconducting magnetic bearings simulation using an H-formulation finite element model," Supercond. Sci. Technol., vol. 31, no. 8, Art. no. 084001 , Jun. 2018

[31] M. D. Ainslie et al., "A new benchmark problem for electromagnetic modelling of superconductors: the high- $T_{\mathrm{c}}$ superconducting dynamo," $\mathrm{Su}$ percond. Sci. Technol., vol. 33, no. 10, Art. no. 105009, Oct. 2020.

[32] L. Queval et al., "Optimization of the superconducting linear magnetic bearing of a Maglev vehicle," IEEE Trans. Appl. Supercond., vol. 26, no. 3, Art. no. 3601905, Apr. 2016.

[33] Y. B. Kim, C. F. Hempstead and A. R. Strnad, "Critical persistent currents in hard superconductors", Phys. Rev. Lett., vol. 9, no. 7, pp. 306-309, Oct. 1962. 